\title{
EQUIVARIANT VECTOR BUNDLES ON COMPLETE SYMMETRIC VARIETIES OF MINIMAL RANK
}

\author{
INDRANIL BISWAS, S. SENTHAMARAI KANNAN, AND D. S. NAGARAJ
}

\begin{abstract}
Let $X$ be the wonderful compactification of a complex symmetric space $G / H$ of minimal rank. For a point $x \in G$, denote by $Z$ be the closure of $B x H / H$ in $X$, where $B$ is a Borel subgroup of $G$. The universal cover of $G$ is denoted by $\widetilde{G}$. Given a $\widetilde{G}$ equivariant vector bundle $E$ on $X$, we prove that $E$ is nef (respectively, ample) if and only if its restriction to $Z$ is nef (respectively, ample). Similarly, $E$ is trivial if and only if its restriction to $Z$ is so.
\end{abstract}

\section{INTRODUCTION}

Let $\sigma$ be an involution of a semisimple adjoint type algebraic group $G$ over $\mathbb{C}$, and let $H=G^{\sigma}$ be the corresponding fixed point locus. De Concini and Procesi constructed a smooth projective variety

$$
X=\overline{G / H}
$$

equipped with an action of $G$, that contains an open dense $G$-orbit $G / H[\mathrm{DP}]$. This $X$ is known as the wonderful compactification of the symmetric space $G / H$.

Richardson and Springer described the $B$-orbits in $G / H$ in terms of the combinatorics of the Weyl group $W$, where $B$ is a Borel subgroup of $G$ (see [RS]). The rank of $G / H$ is defined by Panyushev [Pa] and Knop [Kn1]. The minimal rank symmetric spaces were introduced by Brion $[\mathrm{Br}$. Brion and Joshua have studied the geometry of the closures in $X$ of the $B$-orbits in $G / H$, whenever $G / H$ is of minimal rank [BJ]. Tchoudjem has also studied the closures in $X$ of the $B$-orbits in $G / H$, whenever $G / H$ is of minimal rank [TC].

This paper deals with the restriction of equivariant vector bundles on $X$ to some natural class of subvarieties of $X$, like $B$-orbit closures.

Let $\widetilde{G}$ be the simply connected covering of $G$. The action of $G$ on $X$ produces an action of $\widetilde{G}$ on $X$ using the natural projection $\widetilde{G} \longrightarrow G$. Given an algebraic vector bundle $E$ on $X$, we can get a class of vector bundles on $X$ by pulling back $E$ using the automorphisms of $X$ given by the action of $G$. It can be shown that the isomorphism classes of these pullbacks remain constant if and only if $E$ admits a $\widetilde{G}$-equivariant structure (meaning the action of $\widetilde{G}$ on $X$ admits a lift to an action on $E$ ).

We prove the following (see Theorem 3.5):

1991 Mathematics Subject Classification. 14F17.

Key words and phrases. Wonderful compactification, minimal rank, equivariant bundles, nefness. 
Theorem 1.1. Assume that $G / H$ is of minimal rank. Fix a point $x \in G$. Let $Z$ be the closure of $B x H / H$ in $X$. Let $E$ be a $\widetilde{G}$ equivariant vector bundle on $X$. Then, $E$ is nef (respectively, ample) if and only if the restriction of $E$ to $Z$ is nef (respectively, ample). Similarly, $E$ is trivial if and only if its restriction to $Z$ is trivial.

In [HMP, a similar result is proved for vector bundles on toric varieties.

Before stating the next result, we recall that for the conjugation action of $\widetilde{G}$ on itself, Steinberg proved that for a maximal torus $T$ of $G$, the restriction homomorphism

$$
\mathbb{C}[\widetilde{G}]^{\widetilde{G}} \longrightarrow \mathbb{C}[\widetilde{T}]^{W(G, T)}
$$

is an isomorphism, where $\widetilde{T}$ is the inverse image of $T$ in $\widetilde{G}$ and $W(G, T)$ is the Weyl group of $G$ with respect to $T$ St1]. Hence, we have the Steinberg map

$$
\tau: \widetilde{G} \longrightarrow \widetilde{T} / W(G, T)=\mathbb{A}^{n} .
$$

Let $c$ be a Coxeter element in the Weyl group $W(G, T)$, and let $F$ be the fiber of the Steinberg map $\tau$ containing a representative $n_{c}$ of $c$ in $N_{\widetilde{G}}(\widetilde{T})$. Let $F^{\prime}$ be the image of $F$ in $G$. Set $\mathcal{Z}=Z_{1} \cup Z_{2}$, where $Z_{1}$ is the closure of $F^{\prime}$ in the wonderful compactification $\bar{G}$ of $G$, and $Z_{2}$ is the unique closed $G \times G$ orbit in $\bar{G}[\mathrm{DP}]$.

The group $\widetilde{G} \times \widetilde{G}$ acts on $\bar{G}$ which factors through the action of $G \times G$ on $\bar{G}$. Given an algebraic vector bundle $E$ on $\bar{G}$, the isomorphism classes of its translates by the elements of $G \times G$ remain constant if and only if $E$ admits a $\widetilde{G} \times \widetilde{G}$-equivariant structure.

We also prove the following (see Theorem 4.2):

Theorem 1.2. Let $E$ be a $\widetilde{G} \times \widetilde{G}$ equivariant vector bundle on $\bar{G}$. Then, $E$ is nef (respectively, ample) if and only if the restriction of $E$ to $\mathcal{Z}$ is nef (respectively, ample). Similarly, $E$ is trivial if and only if $\left.E\right|_{\mathcal{Z}}$ is trivial.

\section{PRELIMINARIES}

2.1. Lie algebras and Algebraic groups. In this subsection we recall some basic facts and notation on Lie algebras and algebraic groups (see [Hu, $\mathrm{Hu} 1$ for details). Throughout $G$ denotes a semisimple adjoint-type algebraic group over the field $\mathbb{C}$ of complex numbers. In particular, the center of $G$ is trivial. For a maximal torus $T$ of $G$, the group of all characters of $T$ will be denoted by $X(T)$. The normalizer of $T$ in $G$ will be denoted by $N_{G}(T)$, while

$$
W(G, T):=N_{G}(T) / T
$$

is the Weyl group of $G$ with respect to $T$. Let $R \subset X(T)$ be the root system of $G$ with respect to $T$. For a Borel subgroup $B$ of $G$ containing $T$, let $R^{+}(B)$ denote the set of positive roots determined by $T$ and $B$. Further,

$$
S=\left\{\alpha_{1}, \cdots, \alpha_{n}\right\}
$$


denotes the set of simple roots in $R^{+}(B)$. For $\alpha \in R^{+}(B)$, let $s_{\alpha} \in W(G, T)$ be the reflection corresponding to $\alpha$. The Lie algebras of $G, T$ and $B$ will be denoted by $\mathfrak{g}, \mathfrak{t}$ and $\mathfrak{b}$ respectively. The dual of the real form $\mathfrak{t}_{\mathbb{R}}$ of $\mathfrak{t}$ is $X(T) \otimes \mathbb{R}=\operatorname{Hom}_{\mathbb{R}}\left(\mathfrak{t}_{\mathbb{R}}, \mathbb{R}\right)$.

The positive definite $W(G, T)$-invariant form on $H_{\mathbb{R}}\left(\mathfrak{t}_{\mathbb{R}}, \mathbb{R}\right)$ induced by the Killing form on $\mathfrak{g}$ is denoted by $(-,-)$. We use the notation

$$
\langle\nu, \alpha\rangle:=\frac{2(\nu, \alpha)}{(\alpha, \alpha)} .
$$

In this setting one has the Chevalley basis

$$
\left\{x_{\alpha}, h_{\beta} \mid \alpha \in R, \beta \in S\right\}
$$

of $\mathfrak{g}$ determined by $T$. For a root $\alpha$, we denote by $U_{\alpha}$ (respectively, $\mathfrak{g}_{\alpha}$ ) the one-dimensional $T$ stable root subgroup of $G$ (respectively, the subspace of $\mathfrak{g}$ ) on which $T$ acts through the character $\alpha$.

Let $\sigma$ be an algebraic automorphism of $G$ of order two. Let $H=G^{\sigma}$ be the subgroup consisting of all fixed points of $\sigma$ in $G$. The connected component of $H$ containing the identity element will be denoted by $H^{0}$. We refer to [Ri] and [RS] for following facts.

A torus $T^{\prime}$ of $G$ is said to be $\sigma$-anisotropic if $\sigma(t)=t^{-1}$ for every $t \in T^{\prime}$. Recall that the rank of $G / H$ is defined to be the dimension of a maximal dimensional anisotropic torus.

If $T$ is a $\sigma$-stable maximal torus of $G$, then $\sigma$ induces an automorphism of $X(T)$ of order two. Note that we have $\sigma(R)=R$. Further, one has $T=T_{1} T_{2}$, where $T_{1}$ is a torus such that $\sigma(t)=t$ for every $t \in T_{1}$, and $T_{2}$ is a $\sigma$-anisotropic torus. Clearly $T_{1} \bigcap T_{2}$ is finite. Hence, we have $\operatorname{rank}(G / H) \geq \operatorname{rank}(G)-\operatorname{rank}(H)$.

Throughout, we assume that $G / H$ is of minimal rank, or in other words

$$
\operatorname{rank}(G / H)=\operatorname{rank}(G)-\operatorname{rank}(H) .
$$

We refer to $[\mathrm{Br}]$ and $[\mathrm{Kn} 2]$ for facts about minimal rank.

The following lemma may be known, but for the sake of completeness we provide a proof here.

\section{Lemma 2.2.}

(1) Any two $\sigma$ stable maximal tori of $G$ are conjugate by an element of the connected component $H^{0}$ of $H$ containing the identity element.

(2) Any maximal torus $S$ of $H^{0}$ is contained in a unique maximal torus $T$ of $G$. Further, this $T$ is $\sigma$ stable.

(3) Any Borel subgroup $Q$ of $H^{0}$ is contained in a $\sigma$ stable Borel subgroup $B$ of $G$. Further, the Borel subgroup of $G$ containing $Q$ is unique.

Proof. Proof of (1). Let $T_{1}$ and $T_{2}$ be two $\sigma$ stable maximal tori in $G$. Define

$$
S_{i}:=\left(T_{i} \cap H\right)^{0},
$$


$i=1,2$. Since $G / H$ is of minimal rank, $S_{1}$ and $S_{2}$ are maximal tori in $H^{0}$. Hence, there is an element $h \in H^{0}$ such that $h S_{1} h^{-1}=S_{2}$. Also, $T_{i}=C_{G}\left(S_{i}\right)$ (see [Ri, p. 295, Lemma 5.3 and Lemma 5.4]).

Proof of (2). Take $T=C_{G}(S)$.

Proof of (3). We will first prove the existence of a stable Borel subgroup containing $Q$.

By [St2, p. 51, Lemma 7.5], there is a $\sigma$ stable Borel subgroup $B^{\prime}$ of $G$. By [Ri, p. 295, Lemma 5.1], the intersection $\left(B^{\prime} \cap H\right)^{0}$ is a Borel subgroup of $H^{0}$. Hence, there is a $h \in H^{0}$ such that $Q=h\left(B^{\prime} \cap H\right)^{0} h^{-1}$. Now take $B=h B^{\prime} h^{-1}$.

To prove the uniqueness of $B$, let $B_{1}$ be a Borel subgroup of $G$ containing $Q$. As shown above, there is a $\sigma$ stable Borel subgroup $B$ of $G$ containing $Q$. Choose a maximal torus $S$ of $H^{0}$ lying in $Q$. From part (2) of the lemma we know that $T=C_{G}(S)$ is the unique maximal torus of $G$ containing $S$. Hence, $T$ is contained in both $B_{1}$ and $B$. Thus, there is a $w \in W(G, T)$ such that $w B w^{-1}=B_{1}$.

We now prove that $R^{+}\left(B_{1}\right)=R^{+}(B)$. Let $\alpha \in R^{+}(B) \backslash R^{+}(B)^{\sigma}$. Then the $\sigma$ invariant vector $x_{\alpha}+\sigma\left(x_{\alpha}\right)$ is in the Lie algebra of $Q$. Hence, $x_{\alpha}+\sigma\left(x_{\alpha}\right)$ is in the Lie algebra of $B_{1}$. Thus, both $\alpha$ and $\sigma(\alpha)$ are in $R^{+}\left(B_{1}\right) \backslash R^{+}\left(B_{1}\right)^{\sigma}$. Hence, we have

$$
R^{+}(B) \backslash R^{+}(B)^{\sigma}=R^{+}\left(B_{1}\right) \backslash R^{+}\left(B_{1}\right)^{\sigma}
$$

Now, let $\alpha \in R^{+}(B)^{\sigma}$. We will show that $\sigma$ acts trivially on $U_{\alpha}$. Let $T_{\alpha} \subset T$ be the connected component, containing the identity element, of the kernel of $\alpha$. Consider the restriction of $\sigma$ to $C_{G}\left(T_{\alpha}\right)$. Let $C^{\prime}$ be the commutator subgroup of $C_{G}\left(T_{\alpha}\right)$. If the action of $\sigma$ on $U_{\alpha}$ is not trivial, it follows that there is a one-dimensional $\sigma$ stable anisotropic torus $S^{\prime}$ in $C^{\prime}$. Let $T_{1}=S^{\prime} T_{\alpha}$. Then we have $T_{1}^{\sigma}=T_{\alpha}^{\sigma}$. Hence by [Ri, Lemma 5.4] we have $T_{1}=C_{G}\left(T_{\alpha}^{\sigma}\right)$. But this contradicts the fact that $T_{\alpha}^{\sigma}$ is a singular torus. Hence we have $U_{\alpha} \subset(B)^{\sigma}=Q \subset B_{1}$.

Thus, we have shown that $R^{+}(B)=R^{+}\left(B_{1}\right)$. Hence, we have $B=B_{1}$. This completes proof.

2.3. Nef vector bundle. Let $E$ be an algebraic vector bundle over a complex projective variety $Y$. Let $\mathbb{P}(E)$ denote the associated projective bundle over $Y$ whose fiber over any point $y \in Y$ is the space of all one-dimensional quotients of the fiber $E_{y}$ of $E$ over $y$. The line bundle over $\mathbb{P}(E)$ whose fiber over any one-dimensional quotient is the one-dimensional quotient itself, will be denoted by $\mathcal{O}_{\mathbb{P}(E)}(1)$.

A line bundle $L$ over $Y$ is called nef if for every pair $(C, \varphi)$, where $C$ is an irreducible smooth complex projective curve and $\varphi: C \longrightarrow Y$ is a morphism, the degree of the pullback $\varphi^{*} L$ is nonnegative. A vector bundle $E \longrightarrow Y$ is called nef if the above line bundle $\mathcal{O}_{\mathbb{P}(E)}(1)$ over $\mathbb{P}(E)$ is nef. 


\section{RESTRICTION OF EQUIVARIANT VECTOR BUNDLES TO B-ORBIT CLOSURE}

Let $T$ be a $\sigma$ stable maximal torus of $G$. Let $B$ be a Borel subgroup of $G$ containing $T$ such that for any root $\alpha \in R^{+}(B)$, either $\sigma(\alpha)=\alpha$ or $\sigma(\alpha) \in-R^{+}(B)$.

Let

$$
X:=\overline{G / H}
$$

be the wonderful compactification of the symmetric space $G / H$ constructed in [DP]. Let $Z$ be the closure in $X$ of the $B$-orbit of a point in $G / H$.

Let $P$ be the parabolic subgroup of $G$ containing $B$ such that the $G / P$ is the unique closed $G$ orbit in $X$ (see $[\mathrm{DP})$. In this case, $\sigma(P)$ is opposite to $P$ and $P \bigcap \sigma(P)$ is the Levi subgroup $L$ of $P$. Let $R(L)$ denote the roots of $L$ with respect to $T$.

The following lemma is about a $B$-orbit in $G / H$. We refer to [RS] for information on $B$-orbit closures in $G / H$. For any algebraic group acting on variety, it is well known that there is always a closed orbit. For instance, any orbit of minimal dimension is closed (see, [Hu1, p. 60. Proposition]).

Lemma 3.1. Let $x \in G$ be such that $B \cdot x H / H$ is closed in $G / H$. Then, $x^{-1} B x$ is $\sigma$ stable and there is a $w \in W(G, T)$ such that $B \cdot x H / H=B \cdot w H / H$.

Proof. Let $Q:=\left(x^{-1} B x \bigcap H\right)^{0}$. Since $B \cdot x H / H$ is closed in $G / H$, this $Q$ is a Borel subgroup of $H^{0}$. Further, we have $Q \subset x^{-1} B x$. Hence, by Lemma 2.2, $x^{-1} B x$ is $\sigma$ stable.

Now, let $S=(T \cap H)^{0}$. Since $G / H$ is of minimal rank, this $S$ is a maximal torus in $H^{0}$, and hence we choose a Borel subgroup $Q^{\prime}$ of $H^{0}$ containing $S$. Thus, there is a $h \in H^{0}$ such that $h Q h^{-1}=Q^{\prime}$. Consequently, $h x^{-1} B x h^{-1}$ is a Borel subgroup of $G$ containing $T$. Thus, there is a $w \in W(G, T)$ and a $b \in B$ such that $x h^{-1}=b w$, and we have $B \cdot x H / H=B \cdot w H / H$.

An interesting fact in case of minimal rank is the following uniqueness of the closed $B$-orbit (see, [Re, p. 1788, Proposition 2.2]).

Lemma 3.2. There is a unique closed $B$-orbit in $G / H$.

Proof. Clearly, there is a minimal dimensional $B$-orbit in $G / H$ and it is closed. For its uniqueness, let $B x_{1} H / H$ and let $B x_{2} H / H$ be two closed $B$-orbits in $G / H$. Then, by Lemma 3.1, there are $w_{1}$ and $w_{2}$ in $W$ such that $B \cdot x_{i} H / H=B \cdot w_{i} H / H$ for $i=1,2$.

Let $S=(T \bigcap H)^{0}$. Set $B_{i}:=w_{i}^{-1} B w_{i}$, and $Q_{i}=\left(B_{i} \bigcap H\right)^{0}$ for $i=1,2$. Both $Q_{1}$ and $Q_{2}$ are Borel subgroups of $H^{0}$ containing $S$. Therefore, there is a $\phi \in W\left(H^{0}, S\right)$ such that $\phi Q_{1} \phi^{-1}=Q_{2}$. Hence both $\phi B_{1} \phi^{-1}$ and $B_{2}$ are Borel subgroups of $G$ containing $Q_{2}$. By Lemma 2.2, we have $\phi B_{1} \phi^{-1}=B_{2}$, and hence $w_{1}=w_{2} \phi$. Thus $B x_{1} H / H=$ $B x_{2} H / H$.

We now recall from [BJ] a result of Brion and Joshua. 
Lemma 3.3 ([BJ, p. 482, Lemma 2.1.1]). Let $Y$ be the closure of $T H / H$ in $X$, and let $z$ denote the unique $B$-fixed point in $X$. Then, every $T$ stable curve in $X$ is one of the following:

(1) There is a positive root $\alpha \in R^{+}(B) \backslash R^{+}(L)$ and an element $\phi \in W(G, T)$ such that $\phi(C)=C_{\alpha}=\overline{U_{\alpha} s_{\alpha} z}$. In this case $\alpha$ and $\sigma(\alpha)$ are orthogonal, and $s_{\alpha} s_{\sigma(\alpha)}$ is in $W\left(H^{0},(T \cap H)^{0}\right)$.

(2) There is a restricted root $\gamma=\alpha-\sigma(\alpha)$ and an element $\phi \in W(G, T)$ such that $\phi(C)=C_{z, \gamma}$, where $C_{z, \gamma}$ is the unique T-stable curve containing $z$ and on which $T$ acts through the character $\gamma$. Moreover, the curve $C_{z, \gamma}$ lies in $Y$.

Lemma 3.4. Take $x \in G$, and let $Z$ be the closure of $B x H / H$ in $X$. Then every irreducible $T$ stable curve in $X$ lies in $W(G, T) \cdot Z$.

Proof. Note that the closure of $B \cdot x H / H$ in $G / H$ contains a closed $B$ orbit. Therefore we assume that $B \cdot x H / H$ is the unique closed $B$ orbit in $G / H$.

By Lemma 3.1, there is an element $w \in W(G, T)$ such that $B \cdot x H / H=B \cdot w H / H$. Let $C$ be an irreducible $T$ stable curve in $X$. By Lemma 3.3,

- either there is a positive root $\alpha \in R^{+}(B) \backslash R(L)$ and a $\phi \in W(G, T)$ such that $\phi(C)=C_{\alpha}=\overline{U_{\alpha} s_{\alpha} z}$,

- or there is a restricted root $\gamma$ and a $\phi \in W(G, T)$ such that $\phi(C)=C_{z, \gamma}$.

Recall that $Y=\overline{T H / H}$ and $S=(T \cap H)^{0}$. Now, since $s_{\alpha} s_{\sigma(\alpha)} \in W\left(H^{0}, S\right)$, and $z \in Y$ (see, Lemma $3.3(2)$ ), we have

$$
s_{\alpha} s_{\sigma(\alpha)} \cdot z \in Y
$$

Hence, $w s_{\alpha} s_{\sigma(\alpha)} \cdot z \in w \cdot Y=\overline{T w H / H}$. Since $\alpha$ and $\sigma(\alpha)$ are orthogonal, $s_{\alpha} s_{\sigma(\alpha)}(\alpha)=-\alpha$. Hence, either $w(\alpha)$ is positive or $w s_{\alpha} s_{\sigma(\alpha)}(\alpha)=w(-\alpha)$ is positive. Further, $s_{\alpha} s_{\sigma(\alpha)} \in$ $W\left(H^{0}, S\right)$. Hence $B w H / H=B w s_{\alpha} s_{\sigma(\alpha)} H / H$.

Now, if $w(\alpha)$ is positive, then $U_{w(\alpha)} w s_{\alpha} s_{\sigma(\alpha)} \cdot z$ is contained in $\overline{B w H / H}$. Hence,

$$
w s_{\sigma(\alpha)}\left(C_{\alpha}\right)=\overline{w s_{\sigma(\alpha)} U_{\alpha} s_{\alpha} \cdot z}=\overline{U_{w(\alpha)} w s_{\alpha} s_{\sigma(\alpha)} \cdot z}
$$

is contained in $\overline{B w H / H}$.

If $w s_{\alpha} s_{\sigma(\alpha)}(\alpha)=w(-\alpha)$ is positive, then $w s_{\alpha}\left(C_{\alpha}\right)=\overline{U_{w(-\alpha)} w \cdot z}$ is contained in $\overline{B w H / H}$.

Thus, in either case, the curve $C_{\alpha}$ lies in $W(G, T) \cdot Z$.

Since $C_{z, \gamma} \subset Y$, we have $w\left(C_{z, \gamma}\right) \subset \overline{T w H / H}$. Hence, both type of curves in Lemma 3.3 lie in the union of the $W(G, T)$ translates of $\overline{B w H / H}=\overline{B x H / H}$. This completes the proof. 
Notation: Let $G$ be a semi-simple adjoint group over the field $\mathbb{C}$ of complex numbers as above, and let $\widetilde{G}$ be its universal cover. For a maximal torus $T$ in $G$, we denote its inverse image in $\widetilde{G}$ by $\widetilde{T}$.

Note that $\widetilde{G}$ acts on $X$ and hence we can consider $\widetilde{G}$ equivariant vector bundles on $X$.

Theorem 3.5. Fix a point $x \in G$. Let $Z$ be the closure of $B x H / H$ in $X$, where $B$ is a $\sigma$ stable Borel subgroup of $G$. Let $E$ be a $\widetilde{G}$ equivariant vector bundle on $X$. Then, $E$ is nef (respectively, ample) if and only if the restriction of $E$ to $Z$ is nef (respectively, ample). Similarly, $E$ is trivial if and only if its restriction to $Z$ is trivial.

Proof. Since the restriction of a nef or ample or trivial vector bundle to a subvariety is nef or ample or trivial respectively, we have only to prove the "if" part of the theorem.

First assume that the restriction $\left.E\right|_{Z}$ is nef. We need to show that for any irreducible closed curve $C$ in $\mathbb{P}(E)$, the degree of the line bundle $\left.\mathcal{O}_{\mathbb{P}(E)}(1)\right|_{C}$ is nonnegative, where $\mathcal{O}_{\mathbb{P}(E)}(1) \longrightarrow \mathbb{P}(E)$ is the line bundle defined in Section 2.3 .

Let $Y(\widetilde{T})$ denote the group of all one-parameter subgroups of $\widetilde{T}$, where $\widetilde{T}$, as before, is the inverse image in $\widetilde{G}$ of a $\sigma$ stable maximal torus $T$ of $G$ lying in $B$. Choose a $\mathbb{Z}$-basis $\left\{\lambda_{1}, \lambda_{2}, \cdots, \lambda_{n}\right\}$ of $Y(\widetilde{T})$.

Let $\widetilde{C}$ be an irreducible closed curve in the projective bundle $\mathbb{P}(E)$ over $X$. If the image of $C$ in $X$ is a point, then the degree of $\mathcal{O}_{\mathbb{P}(E)}(1)$ restricted to $\widetilde{C}$ is positive, because $\mathcal{O}_{\mathbb{P}(E)}(1)$ is relatively ample. Hence we can assume that image of $\widetilde{C}$ in $X$ is a curve $C$. Let $\widetilde{C}_{1}$ be the flat limit of $\lambda_{1}(t) \widetilde{C}$ as $t$ goes to zero (i.e., the one dimensional cycle corresponding to the limit point in the Hilbert Scheme of $\mathbb{P}(E)$ ). Then $\widetilde{C}_{1}$ is a 1-dimensional cycle in $\mathbb{P}(E)$ linearly equivalent to $\widetilde{C}$, and the image $C_{1}$ of $\widetilde{C}_{1}$ in $X$ is invariant under $\lambda_{1}$. Inductively, define $\widetilde{C}_{i}$ to be the flat limit of $\lambda_{i}(t) \widetilde{C}_{i-1}$ as $t$ tends to zero, where $2 \leq i \leq n$. Then $\widetilde{C}_{i}$ is linearly equivalent to $\widetilde{C}$, and the image $C_{i}$ of $\widetilde{C}_{i}$ in $X$ is invariant under the action on $X$ of the sub-torus of $T$ generated by the images of $\left\{\lambda_{1}, \lambda_{2}, \cdots, \lambda_{i}\right\}$.

In particular, $\widetilde{C}_{n}$ is linearly equivalent to $\widetilde{C}$, and every irreducible component of $\widetilde{C}_{n}$ lies in the preimage of the $T$ invariant curve $C_{n} \subset X$. But $C_{n}$ can be conjugated to a curve in $Z$ (see Lemma 3.4), hence, by our assumption, $\left.E\right|_{C_{n}}$ is nef. Therefore, the degree of the line bundle $\left.\mathcal{O}_{\mathbb{P}(E)}(1)\right|_{\widetilde{C}}$ is nonnegative (recall that degree $\left(\left.\mathcal{O}_{\mathbb{P}(E)}(1)\right|_{\widetilde{C}}\right)=\operatorname{degree}\left(\left.\mathcal{O}_{\mathbb{P}(E)}(1)\right|_{\widetilde{C}_{n}}\right)$ ). This proves that $E$ is nef.

Next assume that $\left.E\right|_{Z}$ is ample.

For any positive integer $n$, let $\operatorname{Sym}^{n}(E)$ denote the $n$-th symmetric power of the equivariant vector bundle $E$. To prove that $E$ is ample, we first note that there are only finitely many $T$ stable curves in $X$, and all of them lie in $W(G, T) \cdot Z$ (see Lemma 3.4). Thus the assumption implies that $\left.\operatorname{Sym}^{n}(E)\right|_{C}$ is ample for any $T$ stable curve $C$ in $X$ and for any $n \geq 1$.

Since line bundles on $X$ are equivariant for the $\widetilde{G}$ action on $X$, the vector bundles $\operatorname{Sym}^{n}(E) \otimes L$ are all $\widetilde{G}$ equivariant vector bundles on $X$, where $L$ is any line bundle on 
$X$. Fix an ample line bundle $L$ on $X$, and let $n$ be an integer such that $n>\operatorname{degree}\left(\left.L\right|_{C}\right)$ for every $T$ invariant curve $C$ in $X$. Then it follows from the argument in the first part of the proof of the theorem that $\left.\operatorname{Sym}^{n}(E) \otimes L^{-1}\right|_{Z}$ is nef, and hence $\operatorname{Sym}^{n}(E) \otimes L^{-1}$ is nef. This implies $\operatorname{Sym}^{n}(E)$ is ample and hence $E$ is ample (see, [Ha, p. 67, Proposition 2.4]).

Finally assume that $\left.E\right|_{Z}$ is trivial.

Since $\left.E\right|_{Z}$ is trivial, the dual $\left(\left.E\right|_{Z}\right)^{*}=\left.E^{*}\right|_{Z}$ is also trivial. Note that a trivial vector bundle is nef. Therefore, from the first part of the theorem we conclude that both $E$ and its dual $E^{*}$ are nef. Therefore, by [DPS, p. 311, Theorem 1.18] the vector bundle $E$ admits a filtration of holomorphic subbundles

$$
0=E_{0} \subset E_{1} \subset \cdots \subset E_{\ell-1} \subset E_{\ell}=E
$$

such that each successive quotient $E_{i} / E_{i-1}, 1 \leq i \leq \ell$, admits a unitary flat connection. This implies that $E$ is semistable and $c_{j}(E)=0$ for all $j \geq 1$, where $c_{j}$ is the rational Chern class. Now, by [Si, p. 40, Corollary 3.10] the vector bundle $E$ admits a flat holomorphic connection.

The variety $X$ is simply connected, because it is unirational (see, [Se, p. 483, Proposition 1]). Therefore, any holomorphic vector bundle on $X$ admitting a flat holomorphic connection is a holomorphically trivial vector bundle. In particular, the vector bundle $E$ is trivial.

The proof of first two parts of the above theorem closely follows that of [HMP, p.610, Theorem 2.1].

\section{A special Steinberg fiber}

As before, $G$ be a semisimple adjoint group. Let $T$ be a maximal torus of $G, W(G, T)$ the Weyl group of $G$ with respect to $T$ and $B$ a Borel subgroup of $G$ containing $T$. Let $\widetilde{G}$ be the simply connected covering of $G$, and let $\widetilde{T}$ (respectively, $\widetilde{B}$ ) be the inverse image of $T$ (respectively, $B$ ) in $\widetilde{G}$. Let $c$ be a Coxeter element in $W$. We fix a representative $n_{c}$ of $c$ in $N_{\widetilde{G}}(\widetilde{T})$.

Lemma 4.1. The homomorphism $\phi_{c}: \widetilde{T} \longrightarrow \widetilde{T}$ given by $\phi_{c}(t)=t n_{c} t^{-1} n_{c}^{-1}$ is surjective.

Proof. It is enough to prove that the kernel of $\phi_{c}$ is finite. We can choose a reduced expression $c=s_{\alpha_{1}} s_{\alpha_{2}} \cdots s_{\alpha_{n}}$ for $c$ such that $\left\{\alpha_{1}, \alpha_{2}, \cdots, \alpha_{n}\right\}$ is the set of simple roots labeled in some ordering. Let $\beta_{i}=s_{\alpha_{1}} \cdots s_{\alpha_{i-1}}\left(\alpha_{i}\right)$. Then, the set $\left\{\beta_{1}, \beta_{2}, \cdots, \beta_{n}\right\}$ is the set of positive roots which are made negative by $c^{-1}$.

By [YZ, p. 862, Lemma 2.1], we have $\omega_{i}-c\left(\omega_{i}\right)=\beta_{i}$. Now, let $t$ be an element of the kernel of $\phi_{c}$. Then, $\beta_{i}(t)=1$ for every $i=1,2, \cdots, n$. Hence,

$$
\operatorname{kernel}\left(\phi_{c}\right) \subset \bigcap_{i=1}^{n} \operatorname{kernel}\left(\beta_{i}\right) \text {. }
$$


Since $\left\{\beta_{1}, \beta_{2}, \cdots, \beta_{n}\right\}$ is a basis of the root lattice of $\widetilde{G}$ with respect to $\widetilde{T}$, the kernel of $\phi_{c}$ lies in the center of $\widetilde{G}$. Thus, it is finite.

Now, let $\sigma$ be the involution of $G \times G$ defined by $\sigma(x, y)=(y, x)$. Note that the diagonal subgroup $\Delta(G)$ of $G \times G$ is the subgroup of fixed points, $T \times T$ is a $\sigma$-stable maximal torus of $G \times G$ and $B \times B^{-}$is a Borel subgroup having the property that $\sigma(\alpha) \in-R^{+}\left(B \times B^{-}\right)$ for every $\alpha \in R^{+}\left(B \times B^{-}\right)$.

Let $\bar{G}$ denote the wonderful compactification of the group $G$, where $G$ is identified with the symmetric space $(G \times G) / \Delta(G)$.

Now, consider the action of $\widetilde{G}$ on $\widetilde{G}$ by conjugation. We note that $\widetilde{T}$ is stable under the action of $N_{\widetilde{G}}(\widetilde{T})$.

It is proved in St1 that the restriction

$$
\mathbb{C}[\widetilde{G}]^{\widetilde{G}} \longrightarrow \mathbb{C}[\widetilde{T}]^{W(G, T)}
$$

is an isomorphism, and the latter is a polynomial ring. Hence we have the Steinberg map

$$
\tau: \widetilde{G} \longrightarrow \widetilde{T} / W(G, T)=\mathbb{A}^{n} .
$$

Let $F$ be the fiber of the Steinberg map $\tau$ containing a representative $n_{c}$ of $c$ in $N_{\widetilde{G}}(\widetilde{T})$. By an abuse of notation, we denote by $n_{c}$ the image of $n_{c}$ in $N_{G}(T)$. Let $F^{\prime}$ be the image of $F$ in $G$, and let $\mathcal{Z}=Z_{1} \cup Z_{2}$, where $Z_{1}$ is the closure of $F^{\prime}$ in $\bar{G}$ and $Z_{2}$ is the unique closed $G \times G$ orbit in $\bar{G}$.

Theorem 4.2. Let $E$ be a $\widetilde{G} \times \widetilde{G}$-equivariant vector bundle on $\bar{G}$. Then, $E$ is nef (respectively, ample) if and only if the restriction of $E$ to $\mathcal{Z}$ is nef (respectively, ample). Similarly, $E$ is trivial if and only if its restriction to $\mathcal{Z}$ is so.

Proof. Set $W=W(G, T)$. By the proof of Theorem 3.5, it is sufficient to prove that every $T \times T$ stable curve in $\bar{G}$ lies in $(W \times W) \cdot \mathcal{Z}$. It is easy to see that, for every root $\alpha \in R^{+}(B)$, the $T \times T$ stable curve $\overline{\left(\{1\} \times U_{-\alpha}\right) \cdot\left(1, s_{\alpha}\right) \cdot z}$ lies in $Z_{2}$. Similarly, $\overline{\left(U_{\alpha} \times\{1\}\right) \cdot\left(s_{\alpha}, 1\right) \cdot z}$ lies in $Z_{2}$ for every $\alpha \in R^{+}(B)$. Thus, every $T \times T$ stable curve of type 1 in Lemma 3.3 lies in $(W \times W) \cdot \mathcal{Z}$.

On the other hand, by Lemma 4.1, the homomorphism $\phi_{c}$ is onto and hence, the closure of $T n_{c}=\left\{t n_{c} t^{-1} \mid t \in T\right\}$ in $\bar{G}$ is contained in $Z_{1}$. Therefore every $T \times T$ stable curve of type 2 in Lemma 3.3 as well lies in $(W \times W) \cdot \mathcal{Z}$. This completes the proof of the theorem.

\section{ACKNOWLEDGEMENTS}

We thank the referee for helpful comments. The first-named author thanks the Institute of Mathematical Sciences for hospitality while this work was carried out. He also acknowledges the support of the J. C. Bose Fellowship. The second-named author would like to thank the Infosys Foundation for the partial support. 


\section{REFERENCES}

[Br] M. Brion, Construction of equivariant vector bundles, Algebraic groups and homogeneous spaces, 83-111, Tata Inst. Fund. Res., Mumbai, 2007.

[BJ] M. Brion and R. Joshua, Equivariant Chow ring and Chern classes of wonderful symmetric varieties of minimal rank, Transform. Groups 13 (2008), 471-493.

[DP] C. De Concini and C. Procesi, Complete symmetric varieties, Invariant theory Montecatini, 1982, 1-44, Lecture Notes in Math., 996, Springer, Berlin, 1983.

[DPS] J.-P. Demailly, T. Peternell and M. Schneider, Compact complex manifolds with numerically effective tangent bundles, Jour. Alg. Geom. 3 (1994), 295-345.

[Ha] R. Hartshorne, Ample vector bundles, Inst. Hautes Études Sci. Publ. Math. 29 (1966), 63-94.

[HMP] M. Hering, M. Mustata and S. Payne, Positivity properties of toric vector bundles, Ann. Inst. Fourier 60 (2010), 607-640.

$[\mathrm{Hu}] \quad$ J. E. Humphreys, Introduction to Lie algebras and Representation theory, Springer-Verlag, Berlin Heidelberg, New York, 1972.

[Hu1] J. E. Humphreys, Linear Algebraic Groups Representation theory, GTM 21, Springer-Verlag, Berlin Heidelberg, New York, 1975.

[Kn1] F. Knop, Weylgruppe und Momentabbiidung, Invent. Math. 99 (1990), 1-23.

[Kn2] F. Knop, On the set of orbits for a Borel subgroup, Comment. Math. Helv. 70 (1995), 285-309.

[Pa] D.I. Panyushev, Complexity and rank of homogeneous spaces, Dokl. Akad. Nauk SSSR 307 (1989), 276-279.

[Re] N. Ressayre, Spherical homogeneous spaces of minimal rank, Adv. Math. 224 (2010), 1784-1800

[Ri] R. W. Richardson, Orbits, invariants, and representations associated to involutions of reductive groups, Invent. Math. 66 (1982), 287-312.

[RS] R. W. Richardson and T. A. Springer, The Bruhat order on symmetric varieties, Geom. Dedicata 35 (1990), 389-436.

[Se] J.-P. Serre, On the fundamental group of a unirational variety, Jour. Lond. Math. Soc. 34 (1959), 481-484.

[Si] C. T. Simpson, Higgs bundles and local systems, Inst. Hautes Études Sci. Publ. Math. 75 (1992), $5-95$.

[St1] R. Steinberg, Regular elements of semisimple algebraic groups, Inst. Hautes Études Sci. Publ. Math. 25 (1965), 49-80.

[St2] R. Steinberg, Endomorphisms of linear algebraic groups, Memoirs of the American Mathematical Society, No. 80, 1968.

[Tc] A. Tchoudjem, Cohomologie des fibrés en droites sur les variétés magnifiques de rang minimal, Bull. Soc. Math. Fr. 135 (2007), 171-214.

[YZ] S. W. Yang and A. Zelevinsky, Cluster algebras of finite type via Coxeter elements and principal minors, Transform. Groups 13 (2008), 855-895.

Tata Institute of Fundamental Research, Homi Bhabha Road, Mumbai 500004, India

E-mail address: indranil@math.tifr.res.in

Chennai Mathematical Institute, Plot H1, Sipcot it Park, Siruseri, Kelambakam, Chennai 603103, India

E-mail address: kannan@cmi.ac.in

Institute of Mathematical Sciences, C.I.T. Campus, Taramani, Chennai 600113, India

E-mail address: dsn@imsc.res.in 